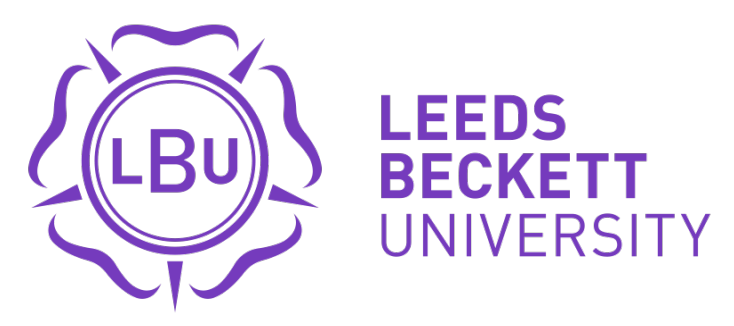

Citation:

Hussain, MA and Sheikh Akbari, A (2018) True colour retrieval from multiple illuminant scene's image. In: IET 3rd International Conference on Intelligent Signal Processing (ISP 2017). IET. ISBN 978-1-78561-707-2, 978-1-78561-708-9 DOI: https://doi.org/10.1049/cp.2017.0350

Link to Leeds Beckett Repository record:

https://eprints.leedsbeckett.ac.uk/id/eprint/4262/

Document Version:

Book Section (Accepted Version)

Creative Commons: Attribution 4.0

The aim of the Leeds Beckett Repository is to provide open access to our research, as required by funder policies and permitted by publishers and copyright law.

The Leeds Beckett repository holds a wide range of publications, each of which has been checked for copyright and the relevant embargo period has been applied by the Research Services team.

We operate on a standard take-down policy. If you are the author or publisher of an output and you would like it removed from the repository, please contact us and we will investigate on a case-by-case basis.

Each thesis in the repository has been cleared where necessary by the author for third party copyright. If you would like a thesis to be removed from the repository or believe there is an issue with copyright, please contact us on openaccess@leedsbeckett.ac.uk and we will investigate on a case-by-case basis. 


\title{
True colour retrieval from multiple illuminant scene's image
}

\author{
*M. A. Hussain, A. Sheikh Akbari \\ School of Computing, Creative Technology and Engineering, Faculty of Arts, Environment and Technology, Leeds Beckett \\ University, U.K., *m.hussein7766@student.leedsbeckett.ac.uk
}

\begin{abstract}
Keywords: colour retrieval, multiple illuminant, segmentation.
\end{abstract}

\begin{abstract}
This paper presents an algorithm to retrieve the true colour of an image captured under multiple illuminant. The proposed method uses a histogram analysis and $\mathrm{K}$-means ${ }^{++}$clustering technique to split the input image into a number of segments. It then determines normalised average absolute difference (NAAD) for each resulting segment's colour component. If the NAAD of the segment's component is greater than an empirically determined threshold. It assumes that the segment does not represent a uniform colour area, hence the segment's colour component is selected to be used for image colour constancy adjustment. The initial colour balancing factor for each chosen segment's component is calculated using the Minkowski norm based on the principal that the average values of image colour components are achromatic. It finally calculates colour constancy adjustment factors for each image pixel by fusing the initial colour constancy factors of the chosen segments weighted by the normalised Euclidian distances of the pixel from the centroids of the selected segments. Experimental results using benchmark single and multiple illuminant image datasets, show that the proposed method's images subjectively exhibit highest colour constancy in the presence of multiple illuminant and also when image contains uniform colour areas.
\end{abstract}

\section{Introduction}

The appearance of a digital image depends on the intrinsic property of the source illuminant, surface reflectance and the camera sensitivity function [1-3]. In a digital image, colours are represented by pixels which are usually get influenced by the colour of the source light [4]. This phenomenon results in unexpected colouring effect that deteriorates the original colour of the object known as colour cast [5]. However, the object's colours do not get saturated if the image is taken under canonical light. Therefore, to retrieve the true colour of an image, a uniform distribution of light spectrum is needed to remove that colour cast so that the image exhibits its true colour under a canonical illuminant [6,7]. A considerable number of colour constancy techniques have been proposed in the literature to attain the original colour of an image [8-13].

The colour constancy techniques algorithms normally estimate the colour of the source illuminant and then predict what the appearance of the scene would be if the source light is canonical. The Grey World [8] method is the simplest and yet widely used techniques that assumes the average reflectance of colour components of an image are achromatic and tends to produce neutral grey. The Max-RGB [9] algorithm, also known as the White Patch technique, which is based on the concept that the maximum response in a scene is caused by a perfect reflectance. This method considers the existence of at least one pixel with maximum reflection of the source illuminant and it integrate the highest values of each colour channel to form that source illuminant. Finlayson and Trezzi [10] proposed the Shades of Grey algorithm to reduce the data dependency of the Grey World and the Max-RGB algorithms. They applied the Minkowski norm as a mathematically rigorous tool to incorporate a constraint on illumination by assigning higher weights to the pixel values. Moreover, they have shown that the max-RGB and the Grey World method are two different instantiations of the Minkowski p-norm (with $p=\infty$ and $p=0$ ). It has been shown that the Shades of Grey algorithm reliably outperforms Grey World or Max-RGB technique, where its pnorm is in the range of 4-6. Van de Weijer et al. [11] showed that a p-norm summary based on image derivatives can deliver improved performance. They proposed the Grey Edge Hypothesis method that assumes the average values of an image derivatives are also achromatic. They have incorporated a smoothing step into their algorithm using a Gaussian filter ( $\sigma=1$ or 2 , where $\sigma$ determines the order of the derivatives) over a larger neighbourhood. Another extension of Grey Edge method was proposed by Gijsenij et al. [12]. This method considers the photometric properties of different kind of edges and their distinctive influences on the performance of the illuminant estimation. A computationally time-effective colour constancy algorithm based on adaptively stretching the histogram information was proposed by Wang et al. [13]. However, such stretching increases intensity of the image, hence deteriorates the performance of the technique in adjusting the colour of pixels in some cases. From the literature it can be found that most of the state of art algorithms assume a uniform light across the scene to estimate the source illuminant. However, in reality, a scene usually lit by multiple light sources. This significantly deteriorates the performance of the state of art algorithms. Therefore, it seems estimating local illuminates in an image is the key for performing colour constancy adjustment. Various approaches for local illuminant estimation were proposed in the literature such as: grid based 
sampling [14] and super pixel based sampling [15]. Such approaches aim to combine the same illuminant pixels together to form a larger patch for estimation, this process may generate too large or small patches, which could affect the colour constancy adjustment. Therefore, an automatic segmentation approach is needed to segment the image into reasonable patch size for more accurate estimation. In addition, there have been a remarkable tendency of using entire image pixels to estimate the source illuminant, while in reality not all image pixels contain relevant information for colour constancy adjustment. This often results, a biased image, toward the colour of the large uniform colour areas.

This paper presents a colour constancy algorithm to retrieve the original colour of the scene's image lit by multiple illuminant. The proposed algorithm applies a histogram analysis on the image data to determine the required number of segments and the uses the K-means ${ }^{++}$algorithm [16] to split the image into segments. It then assess the colour variability of each segment using the normalised average absolute difference (NAAD) value of its colour components. Segments with the NAAD value higher than empirically determined threshold values, assumes to have enough colour variety, are chosen to contribute into whole image colour constancy adjustment. The initial colour constancy for the selected segments' colour components are calculated using the Minkowski-norm. Colour constancy factors for each pixel of the image finally calculated by combining the colour constancy of the chosen segments weighted by normalised Euclidian distance of the pixel from the centroids of the selected pixels. The performance of the proposed technique is assessed using publicly available image datasets. Results show the merit of the proposed method over the state of art techniques. The rest of paper is organised as follows: Section 2 presents the proposed algorithm, Section 3 gives the experimental results and Section 4 conclude the paper.

\section{Proposed Algorithm}

The proposed colour retrieval algorithm contains three key steps including image segmentation, assessment of the colour variability of segments; and generating colour constancy factors for each pixel.

\subsection{Image segmentation}

To determine the number of segments for automatic image segmentation, the proposed algorithm converts the RGB image into a grey image and split the image data into a histogram with 256 bins. It smooths the image data by using a one-dimensional Gaussian filter with the following parameters:

\section{Gaussian Filter $=\left[\begin{array}{lll}0.25 & 0.5 & 0.25\end{array}\right]$}

The number of the local maxima within the resulted smoothed histogram are determined as the number of the segments within the image. In this experiment, the minimum distance between two local maxima and the minimum local maxima height were set to 0.05 and 0.10 percent of the total number of image pixels, respectively. The calculated number of segments and the $L^{*} \mathrm{a}^{*} \mathrm{~b}^{*}$ format of the RGB input image are fed into the Kmean++ algorithm, where $\mathrm{L}^{*}$ carries the lightness and $\mathrm{a}^{*}$ and $\mathrm{b}^{*}$ contain the colour components of the input image. The Kmeans ${ }^{++}$clustering algorithm divides the input image pixels into a number of segments based on their colour properties. The centroid selection and segmentation steps of the Kmeans++ algorithm are as follows:

i. Randomly select the initial $n$ centroids of the segments from $a^{*}$ and $b^{*}$ components of the input image, which are named as $\left(c_{1} \ldots c_{n}\right)_{i=0}$ and assign zero to $\mathrm{i}$, where $i$ represents the current iteration.

ii. $\quad$ Determine the $n$ new centroids, named $\left(c_{1} \ldots c_{n}\right)_{i}$ in Fig. 1, by calculating the average of the coefficients in each resulting segment and increment $i$ variable by one.

iii. Determine the $n$ new centroids, named $\left(\mathrm{c}_{1} \ldots \mathrm{c}_{n}\right)_{i}$, by calculating the average of the coefficients in each resulting segment and increment $i$ variable by one.

iv. Determine the $n$ new centroids, named $\left(c_{1} \ldots c_{n}\right)_{i}$, by calculating the average of the coefficients in each resulting segment and increment $i$ variable by one.

v. Check if $i$ is greater than the predefined number of iteration (noi), if $i$ is greater than noi, the previously determined segments, $\left(c l_{1} \ldots \mathrm{cl}_{n}\right)_{i}$, are the final segments. Re-name them as: $c l_{1} \ldots \mathrm{cl}_{n}$, and the segmenting process is completed; otherwise go back to step ii.

\subsection{Assessment of the colour variability of segments and initial balancing factors calculation}

In this section, the resulting segments are assessed to determine their colour variability. Segments with acceptable colour information are chosen and the Minkowski norm for their colour components calculated and considered as initial colour constancy factors. To assess the colour variability of each segment colour component, the normalised average absolute difference (NAAD) of the colour component is calculated using (2).

$$
N A A D_{C}=\frac{\sum_{(i, j)}\left(\left|c_{S}(i, j)-\bar{M}_{S_{C}}\right|\right.}{\left(N \times \bar{M}_{S_{C}}\right)}
$$

where $N A A D_{C}$ is the Normalized Average Absolute Difference of the component $C$ of the segment, $C_{S}(i, j)$ represents component $C$ 's pixel value at location $i$ and $j, \bar{M}_{S_{C}}$ is the average pixel value of the component $\mathrm{C}$ pixel values and $\mathrm{N}$ is the total number of pixels in the segment. The resulting $N A A D_{C}$ value is compared with empirically pre-determined threshold values for $\mathrm{R}, \mathrm{G}$ and $\mathrm{B}$ components, respectively. If three calculated $N A A D_{C}$ value are greater than their respective threshold values, it implies that the segment contains a nonuniform colour areas. A bit in the decision vector (DV), which was initially set to zero, is set to 1 to represent the chosen 
segment's pixels. As a pre-processing step, extensive experiments were done using the benchmark image datasets to determine the threshold values. It is empirically found that a threshold value of 0.1 for $\mathrm{R}, \mathrm{G}$ and $\mathrm{B}$ component can efficiently alleviate segment with uniform areas.

To give an insight into the normalised average absolute difference (NAAD) values of different image segments, the calculated NAAD's for the sample image from the Grey Ball dataset [17] after applying K-means ${ }^{++}$algorithm, is shown in Table 1. From this table it can be seen that the NAAD value of the segment 4 , which is mainly the uniform area, are lower than the threshold values.

\subsection{Generating colour constancy factors for each pixel}

The proposed algorithm is then calculates initial colour constancy adjustment factors for each of the selected segments as it follows: a) it calculates the Minkowski-norm for each colour component of the segment using equation (3).

$$
M N_{S_{C}}=\left\{\frac{\left(\sum_{(i, j)} C_{S}(i, j)\right)^{p}}{N}\right\}^{1 / p}
$$

where $M N_{S_{C}}$ is the Minkowski-norm value of colour component $\mathrm{C}, C \in\{R, G, B\}$, of the selected segment, $C_{S}(i, j)$ represents component $C$ 's pixel value at location $i$ and $j$ and $p$ is the Minkowski norm value. In this paper the Minkowski norm value was set to $6(p=6)$ based on results presented in [10] that the best performance of the illuminant estimation as a function of the Minkowski norm is achieved at Minkowski norm of 6. b) Initial scaling factors for each colour component of the selected segments are then calculated using equation (4).

$$
K_{C_{S}}=\frac{\overline{M N}_{S_{C}}}{{ }^{M N_{S_{C}}}}
$$

where $K_{C_{S}}$ is the initial scaling factor for colour component $\mathrm{C}$ of the selected segment, $\overline{M N}_{S_{C}}$ is the average of the resulting Minkowski-norm values, $M N_{S_{C}}$ is the Minkowski-norm value of colour component $\mathrm{C}$. The proposed algorithm is then combined the calculated initial colour constancy factors of the selected segments to generate colour constancy factors for each pixel as follows. It first calculates the Euclidian distance of the pixel from the centroids of all selected segments. It then combines the colour constancy weighing factors of all selected segments to generate colour constancy weighting factor for the pixel using equation (5).

$$
\begin{aligned}
& k_{C}=\frac{d_{1}}{d_{1}+d_{2}+\cdots+d_{n}}\left(k_{C 1}\right)+\frac{d_{2}}{d_{1}+d_{2}+\cdots+d_{n}}\left(k_{C 2}\right)+\cdots+ \\
& \frac{d_{n}}{d_{1}+d_{2}+\cdots+d_{n}}\left(k_{C n}\right)
\end{aligned}
$$

where $k_{C}$ is the colour constancy weighting factor for component $\mathrm{C}$ of the pixel, $C \in\{R, G, B\}$, and $\mathrm{d} 1, \mathrm{~d} 2, \ldots, \mathrm{dn}$ are the Euclidian distance of the pixel from the centroid of the segments $1,2, . ., \mathrm{n}$, respectively.

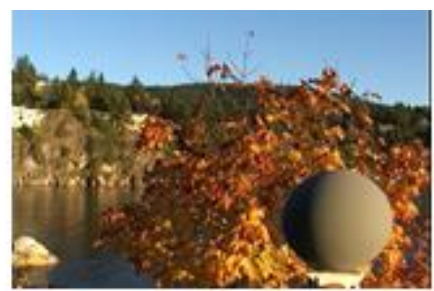

(a)

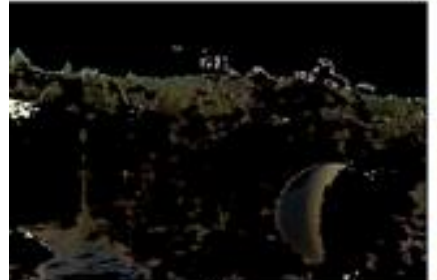

(b)

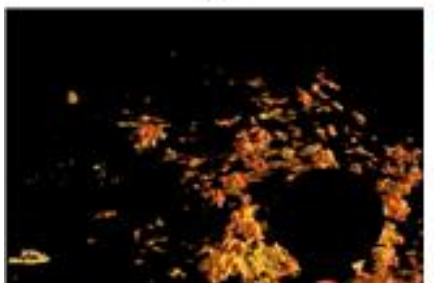

(d)

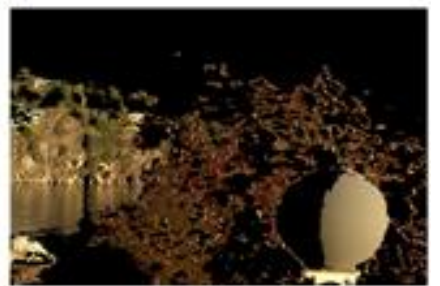

(c)

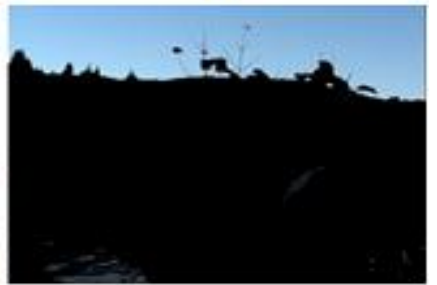

(e)
Figure 1: Application of the propsoed method to an image of the Grey Ball dataset [17], where the input image is divided into four segments and segments 4 has a large uniform colour area.

\begin{tabular}{|c|c|c|c|c|}
\hline & $\begin{array}{c}\text { Segment } \\
\mathbf{1}\end{array}$ & $\begin{array}{c}\text { Segment } \\
\mathbf{2}\end{array}$ & $\begin{array}{l}\text { Segment } \\
\mathbf{3}\end{array}$ & $\begin{array}{c}\text { Segment } \\
\mathbf{4}\end{array}$ \\
\hline $\mathrm{NAAD}_{\mathrm{R}}$ & 0.19 & 0.16 & 0.12 & 0.06 \\
\hline $\mathrm{NAAD}_{\mathrm{G}}$ & 0.21 & 0.22 & 0.21 & 0.05 \\
\hline $\mathrm{NAAD}_{\mathrm{B}}$ & 0.26 & 0.30 & 0.32 & 0.04 \\
\hline
\end{tabular}

Table 1: normalised average absolute difference (NAAD) values of the coefficients within the colour components of the image.

\section{Experimental Results}

The proposed algorithm and the Grey World [8], Max-RGB [9], Shades of Grey [10], 1st Order Grey Edge and 2nd Order Grey Edge [11], Weighted Grey Edge [12] and the Histogram Stretch [13] states of the art colour constancy techniques were applied to 555 images of the 'Granville Island Market-1' of the 
(a)

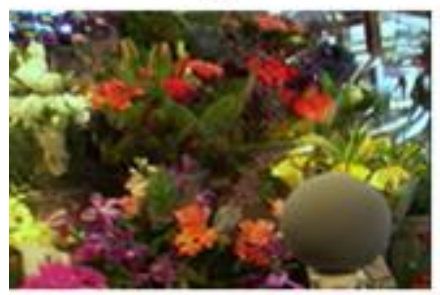

(c)

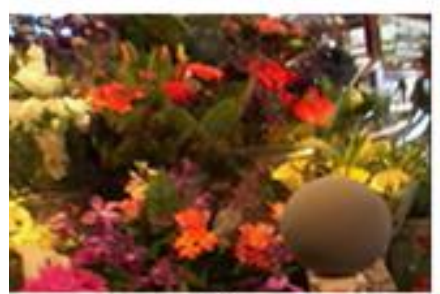

(e)

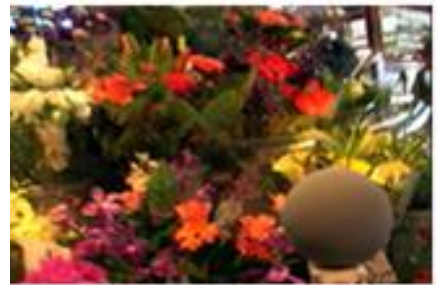

(g)

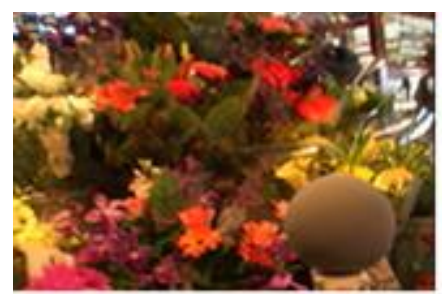

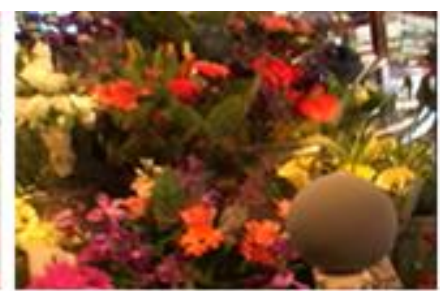

(b)

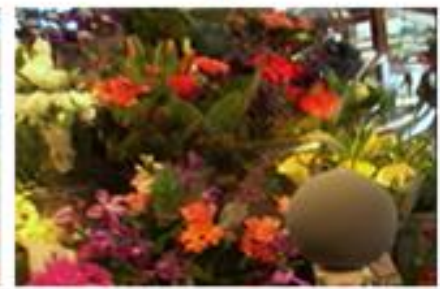

(d)

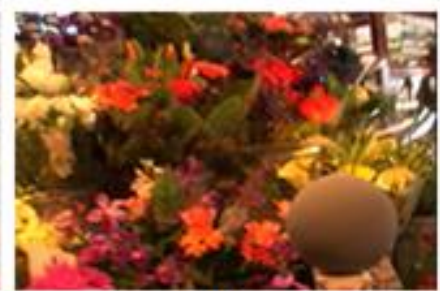

(f)

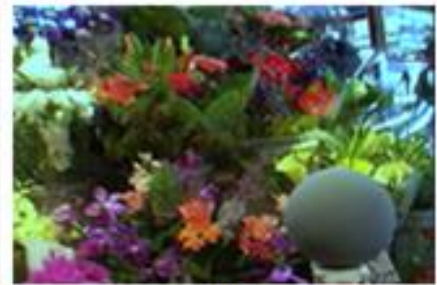

(h)

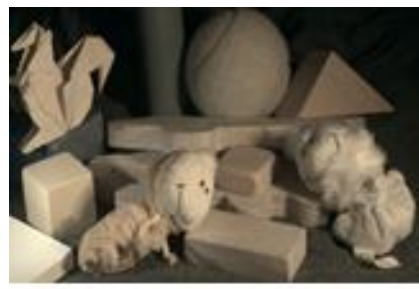

(a)

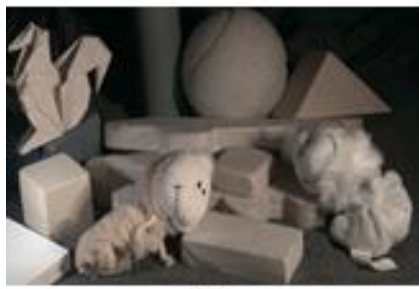

(c)

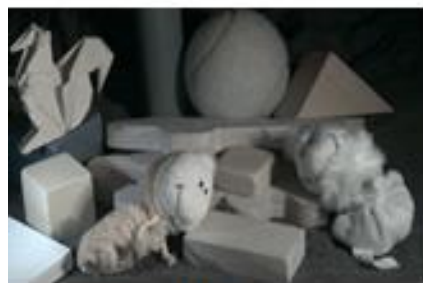

(e)

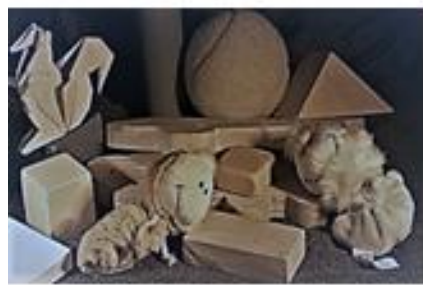

(g)

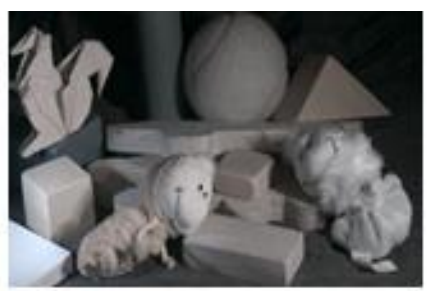

(b)

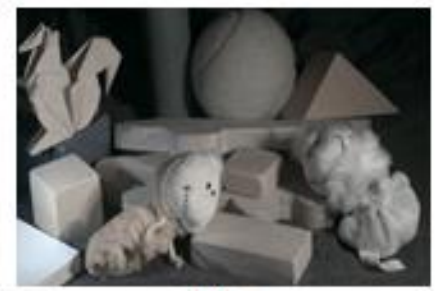

(d)

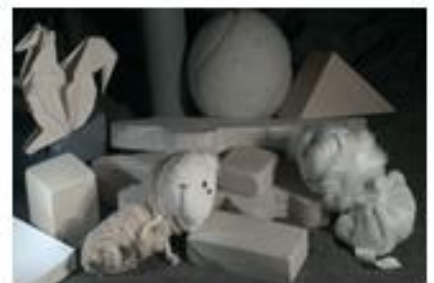

(f)

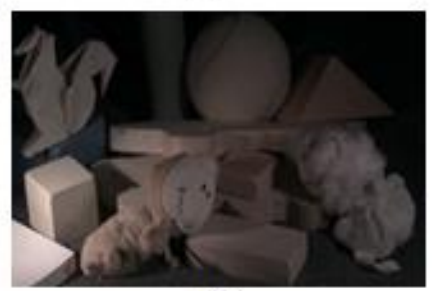

(h)
Figure 2: Original and colour balanced images of sub-set 'Granville Island Market-1' of the Grey Ball dataset: a) Original image, b) Grey World c) Max-RGB, d) Shades of Grey, e) Grey Edge ( $1^{\text {st }}$ Order, f) Grey Edge ( $2^{\text {nd }}$ Order), g) Weighted Grey Edge, and h) Proposed method's image.

Grey Ball dataset [17] and 58 images of the 'laboratory' images of the MIMO dataset [18]. The Grey Ball dataset is the most widely used dataset for colour constancy experiments. It contains images of various scenarios taken under different natural lighting conditions. Its images are of $240 \times 360$ resolution and a grey ball is located in foreground of the scene. The 'laboratory' subset of the MIMO image dataset contains images taken under controlled illuminant settings.

Since human eyes are the final and most reliable judge for assessing the quality of the colour balanced images, so visual appearance of the images was considered to evaluate the performance of the colour constancy techniques. For accuracy, the observations have been done using a 42-inch full high definition screen of $1920 \times 1080$ resolution.

The resulting images show highest subjective colour constancy on most of the images. To give a subjective sense of the achieved colour constancy using the proposed technique, two sample images from the 'Granville Island Market-1' of the Grey Ball dataset and 'laboratory' part of the MIMO dataset
Figure 3: Original and colour balanced images of the MIMO (laboratory) dataset: a) Original image, b) Grey World c) MaxRGB, d) Shades of Grey, e) Grey Edge ( $1^{\text {st }}$ Order, f) Grey Edge ( $2^{\text {nd }}$ Order $), g$ ) Weighted Grey Edge, and h) Proposed method's image.

were taken and colour balanced using the proposed and other above-mentioned techniques. The resulting images are shown in figure 2 and figure 3, respectively. The visual appearance of the grey ball is purely grey in figure 2 and the colour of the objects in figure 3 seems original as the colour cast has been removed by the proposed method. These colour balanced images demonstrate the promising performance of the proposed algorithm.

\section{Conclusions}

This paper presented an algorithm to retrieve the true colour of an image object captured under multiple illuminant scene. Considering the presence of multiple illuminant, it automatically splits the image into a number of segments using histogram analysis and K-means++ algorithm. The colour variability of each segment is assessed by calculating the normalised average absolute difference (NAAD) for each of its colour component. Segments, which have sufficient colour 
variability amongst its pixels, were to be used for the whole image colour constancy adjustment. The Minkowski norm were then used to calculate the initial colour constancy factor for the selected segments. The initial colour constancy of the selected segments was then combined to generate colour constancy factors for each pixel, where they were regulated by the normalised Euclidian distance of the pixel from the centroid of the selected segments. Experimental results using the benchmark dataset showed that the proposed algorithm outperforms the state of the art techniques, subjectively.

\section{References}

[1] D. Fourure, R. Emonet, E. Fromont, D. Muselet, A Trémeau and C. Wolf, "Mixed pooling neural networks for color constancy," IEEE International Conference on Image Processing, Phoenix, AZ, pp. 3997-4001, (2016).

[2] K. Yanlin Qian, J.K. Chen, J. Kämäräinen, J. Nikkanen and J. Matas, "Deep structured-output regression learning for computational color constancy," International Conference on Pattern Recognition, Cancun, pp. 18991904, (2016).

[3] S. Bianco, C. Cusano and R. Schettini, "Color constancy using CNNs," IEEE Conference on Computer Vision and Pattern Recognition Workshops, Boston, MA, pp. 81-89, (2015)

[4] A. Gijsenij and T. Gevers, "Color Constancy using Image Regions," IEEE International Conference on Image Processing, San Antonio, TX, pp. 501-504, (2007).

[5] G. Schaefer, S. Hordley and G. Finlayson, "A combined physical and statistical approach to colour constancy," IEEE Computer Society Conference on Computer Vision and Pattern Recognition, vol. 1, pp. 148-153, (2005).

[6] K. F. Yang, S. B. Gao and Y. J. Li, "Efficient illuminant estimation for color constancy using grey pixels," IEEE Conference on Computer Vision and Pattern Recognition, Boston, MA, pp. 2254-2263, (2015).

[7] J. Im, D. Kim, J. Jung, T. C. Kim and J. Paik, "Dark channel prior-based white point estimation for automatic white balance," IEEE International Conference on Consumer Electronics (ICCE), Las Vegas, NV, pp. 123124, (2014).

[8] G. Buchsbaum. "A spatial processor model for object colour perception", Journal of the Franklin Institute, vol. 310(1), pp. 1-26, (1980).

[9] E. Land. "The retinex theory of colour vision", Scientific American, 237(6), pp. 108-128, (1977).

[10] G.D. Finlayson, E. Trezzi. "Shades of grey and colour constancy", Proc. IS\&T/SID Color Imaging Conf., pp. 37-41, (2004).

[11] J. van de Weijer, T. Gevers, A. Gijsenij. "Edge based colour constancy", IEEE Trans on Image Processing, pp.2 207-2217, (2007).

[12] A. Gijsenij, T. Gevers, J. Van de Weijer. "Improving Color Constancy by Photometric Edge Weighting," IEEE Trans. on Pattern Analysis and Machine Intelligence, 34(5), pp. 918-929, (2012).
[13] S. Wang, Y. Zhang, P. Deng, F. Zhou., "Fast automatic white balancing method by colour histogram stretching," Image and Signal Processing (CISP), vol.2, no., pp.979983, pp.15-17, (2011).

[14] A. Gijsenij, R. Lu, T. Gevers. "Color Constancy for Multiple Light Sources", IEEE Transactions on Image Processing, 21(2), pp. 697-707, (2012).

[15] M. Bleier et al., "Color constancy and non-uniform illumination: Can existing algorithms work?", IEEE International Conference on Computer Vision Workshops, pp. 774-781, (2011).

[16] D. Arthur, and S. Vassilvitskii, "K-means++: The Advantages of Careful Seeding," Proceedings of the Eighteenth Annual ACM-SIAM Symposium on Discrete Algorithms, pp. 1027-1035, (2007).

[17] F. Ciurea, B. Funt. "A Large Image Database for Color Constancy Research", Proceedings of the Imaging Science and Technology Eleventh Color Imaging Conference, pp. 160-164, (2003).

[18] S. Beigpour, C. Riess, J. van de Weijer, and E. Angelopoulou, "Multi-Illuminant Estimation with Conditional Random Fields," in IEEE Transactions on Image Processing, 23(1), pp. 83-96, (2014). 